Revista Destaques Acadêmicos, Lajeado, v. 12, n. 3, 2020. ISSN 2176-3070

DOI: http://dx.doi.org/10.22410/issn.2176-3070.v12i3a2020.2652

http://www.univates.br/revistas

\title{
RELAÇÃO DO GANHO DE PESO NA GESTAÇÃO COM O ESTADO NUTRICIONAL PRÉ-GESTACIONAL E COM O CONSUMO DIETÉTICO
}

\author{
Fernanda Scherer Adami ${ }^{1}$, Michele Dutra Rosolen ${ }^{2}$, Mabel Nilson Alves ${ }^{3}$, \\ Francieli Lays Silva Schedler ${ }^{4}$, Tais Battisti Guerra ${ }^{5}$, Ioná Carreno ${ }^{6}$
}

Resumo. A gravidez provoca modificações metabólicas, nutricionais e fisiológicas no organismo materno, que geram necessidade aumentada de nutrientes, tanto em termo de micro e macronutrientes. Verificar a relação entre o estado nutricional pré-gestacional e ganho de peso com o consumo alimentar em gestantes. Estudo quantitativo transversal realizado com gestantes assistidas pelo serviço de assistência pré-natal em uma Unidade Básica de Saúde. Foi aplicado questionário estruturado e recordatório 24 horas, este foi calculado utilizando o software Avanutri ${ }^{\circledR}$ e os parâmetros utilizados seguiram as Dietary References Intake (DRIs), considerando Recommended Dietary Allowances (RDA) para calorias e Estimated Average Requirement (EAR) para macronutrientes e micronutrientes. Para avaliação do estado nutricional pré-gestacional utilizou-se o Índice de Massa Corporal (IMC). Foram avaliadas 255 gestantes, sendo que $51 \%$ apresentaram ganho de peso acima do recomendado. Observou-se que a faixa etária estava associada ao estado nutricional de maneira que as gestantes menores de 25 anos foram associadas ao baixo peso e à eutrofia e aquelas com 25 anos ou mais ao excesso de peso $(p=0,000)$. O consumo de gordura saturada foi associado ao ganho de peso acima do recomendado $(p=0,013)$. Observou-se consumo abaixo do adequado dos micronutrientes avaliados. Os macronutrientes e micronutrientes não estavam significativamente associadas ao estado nutricional. Conclui-se que o ganho de peso gestacional pode ter influência

1 Nutricionista, Doutora em Ambiente e Desenvolvimente, Docente do Curso de Nutrição Universidade do Vale do Taquari - Univates

2 Nutricionista, Pós-doutoranda em Ciência e Tecnologia de Alimentos, Universidade Federal de Pelotas- UFPel

3 Nutricionista, Doutoranda em Nutrição e Alimentos, Universidade Federal de Pelotas- UFPel

4 Nutricionista, Universidade do Vale do Taquari - Univates

5 Bolsista de Iniciação Científica - Universidade do Vale do Taquari - Univates

6 Enfermeira, Docente do Curso de Enfermagem, Universidade do Vale do Taquari - Univates 
direta do consumo de alimentos ricos em gordura saturada, e por isso a necessidade de intervenções nutricionais individualizadas para prevenir os agravos à saúde das gestantes e seus conceptos.

Palavras-chave: Gravidez; Antropometria; Recomendação nutricional; Consumo alimentar.

\section{Introdução}

A gestação provoca modificações metabólicas, nutricionais e fisiológicas no organismo materno, que geram necessidade aumentada de macronutrientes e micronutrientes. $\mathrm{O}$ inadequado aporte energético pode levar a uma competição entre a mãe e o feto, limitando a disponibilidade dos nutrientes necessários ao adequado crescimento fetal, uma vez que a única fonte de nutrientes do concepto é constituída pelas reservas nutricionais e ingestão alimentar materna (MELO et al., 2007). A inadequação do estado antropométrico materno, tanto pré-gestacional quanto gestacional, se constitui problema de saúde pública, pois favorece o desenvolvimento de intercorrências gestacionais e influencia as condições de saúde do bebê e da mãe (PADILHA et al., 2007), sendo um forte determinante do desfecho da mesma (GIACOMELLO et al., 2008).

O comprometimento na saúde da gestante em decorrência da alimentação inadequada inclui os sintomas de deficiências de micronutrientes (DA SILVA et al; 2007). Por outro lado, o aporte calórico em excesso pode promover ganho de peso gestacional acima do recomendado, que está associado à maior retenção de peso após o parto, sobrepeso e obesidade em mulheres (MARTINS e BENÍCIO, 2011). Conhecer o consumo dietético da população atendida pode melhorar a qualidade das informações prestadas pelos profissionais em seus atendimentos. Os aspectos das recomendações nutricionais podem ser essenciais para fortalecer o planejamento e desenvolvimento saudável da gravidez, minimizando riscos de comprometimento materno e perinatal (FAZIO et al., 2011).

O objetivo deste estudo foi de verificar a relação entre o estado nutricional e consumo dietético de gestantes de um município do interior do Rio Grande do Sul (RS).

\section{Metodologia}

O presente estudo caracteriza-se como quantitativo transversal. Participaram 255 gestantes no período agosto a setembro de 2012 que estavam sendo assistidas pelo serviço de assistência pré-natal em uma Unidade Básica de Saúde (UBS) de um município de 65.946 mil habitantes do RS.

Foram convidadas a participar da pesquisa as gestantes, independente de idade materna e gestacional, que foram atendidas pela UBS no período de coleta de dados. Foram excluídas as que não estavam em dia com o pré-natal e 
depois de informadas dos objetivos do estudo, não concordaram em assinar o Termo de Consentimento Livre Esclarecido.

Foi aplicado um questionário estruturado, contendo as informações socioeconômicas: número de gestações, zona de moradia, renda familiar, número de pessoas que moram na casa, escolaridade, ocupação atual e estado civil. Foram copiados da carteirinha de gestante os dados: idade da gestante, semana gestacional, peso atual, peso pré-gestacional e altura. Foi observado o número de refeições realizadas pelas gestantes, quantidade de água ingerida por dia e uso de sal e gordura adicionados na alimentação mensalmente.

Para avaliação do estado nutricional pré-gestacional utilizou-se o IMC obtido pela relação: peso pré-gestacional $(\mathrm{kg}) /$ [altura $\left(\mathrm{m}^{2}\right)$ ] (coletados da carteirinha de gestante), a classificação do estado nutricional seguiram os parâmetros do World Health Organization (WHO) (WHO, 1990). O ganho ponderal materno foi analisado segundo o estado nutricional antropométrico pré-gestacional pela classificação do Institute of Medicine (IOM) (IOM, 1990).

Os dados sobre o consumo dietético foram obtidos utilizando recordatório de 24 horas, em dois dias diferentes e alternados. Em seguida foi realizada a avaliação da composição química das dietas, utilizando o software Avanutri®. A definição de adequação de energia foi calculada conforme a RDA (Recommended Dietary Allowances, Ingestão Dietética Recomendada) (NRC, 1989). Os macronutrientes e micronutrientes seguiram os critérios da EAR (Estimated Average Requirement, Requerimento Médio Estimado), preconizado pela Dietary Reference Intake (DRI) (DRI, 1998, 2005).

Os resultados foram analisados através dos testes Qui-quadrado e Teste Exato de Fisher. Os resultados foram considerados significativos a um nível de significância de $95 \%(\mathrm{p}<0,05)$ e o software utilizado para esta análise foi o STATA versão 12.0 .

O estudo foi aprovado pelo Comitê de Ética em Pesquisa do Centro Universitário -Univates, sob o número 41575.

\section{Resultados}

Das 255 gestantes estudadas, 82,4\% (210) eram adultas e $17,6 \%$ (45) adolescentes; $39,6 \%$ (101) residiam na zona rural e 60,4\% (154) na zona urbana; $81,9 \%$ (212) apresentavam companheiro e 16,9\% (43) eram solteiras. Quanto à renda familiar, $48,6 \%$ (124) recebiam um salário mínimo ou menos por mês, $33,7 \%$ (86) até dois salários mínimos e 17,7\% (45) três ou mais salários mínimos. Em relação à escolaridade, 60,7\% (155) apresentam o ensino fundamental incompleto e completo, $36 \%$ (92) ensino médio incompleto e completo e $3,2 \%$ (8) relataram ter iniciado o ensino superior.

Quanto ao número de refeições, 46,6\% (119) faziam de 1 a 4 refeições diárias e 53,3\% (136) realizavam 5 ou mais refeições ao dia. Quanto ao consumo de água entre as gestantes $27,1 \%$ (69) tomavam a quantidade suficiente com 
ingestão de mais de 8 copos ao dia e 72,9\% (186) insuficiente, sendo que destes, $10,6 \%$ (27) relataram que não consumiam água; $87,5 \%$ (223) não acrescentavam sal adicional nas refeições. As gestantes referiram que o tipo de gordura utilizada em casa estava sendo banha, óleo vegetal ou ambos, 9\% (23), 78\% (199) e 13\% (33), respectivamente.

Do total das gestantes $29,8 \%$ (76) estavam no primeiro trimestre de gestação, 34,9\% (89) no segundo trimestre e 35,3\% (90) no terceiro. Em relação ao estado nutricional pré-gestacional, 3,9\% (10) estavam com baixo peso, 50,6\% (129) eutróficas, $29,4 \%$ (75) tinham sobrepeso e 16,1\% (41) obesas. E quanto ao ganho de peso até o momento da avaliação, 11,8\% (30) apresentavam-se com ganho de peso insuficiente, $37,3 \%$ (95) tiveram um aumento de peso adequado e 51\% (130) tiveram elevado ganho de peso. Relacionando o estado nutricional pré-gestacional com o ganho de peso acima do recomendado (Tabela 1), verificou-se que o ganho de peso foi maior nas gestantes eutróficas $(47,7 \%)$, seguido das com sobrepeso $(33,1 \%)$, obesidade $(16,9 \%)$ e baixo peso $(2,3 \%)$.

TABELA 1. Comparação do Estado Nutricional Pré Gestacional com a classificação de Ganho de Peso.

\begin{tabular}{|c|c|c|c|c|c|c|c|}
\hline \multirow[b]{3}{*}{ Classificação } & \multicolumn{6}{|c|}{ Classificação do ganho de peso } & \multirow[b]{3}{*}{$\mathrm{P}$} \\
\hline & \multicolumn{2}{|c|}{ Baixo } & \multicolumn{2}{|c|}{ Adequado } & \multicolumn{2}{|c|}{ Acima } & \\
\hline & $\mathrm{N}$ & $\%$ & $\mathrm{~N}$ & $\%$ & $\mathrm{~N}$ & $\%$ & \\
\hline Baixo Peso & 1 & 3,3 & 6 & 6,3 & 3 & 2,3 & 0,557 \\
\hline Eutrofia & 16 & 53,3 & 51 & 53,7 & 62 & 47,7 & \\
\hline Sobrepeso & 7 & 23,3 & 25 & 26,3 & 43 & 33,1 & \\
\hline Obesidade & 6 & 20,0 & 13 & 13,7 & 22 & 16,9 & \\
\hline
\end{tabular}

Comparando o estado nutricional com as demais variáveis, observou-se que somente a faixa etária estava associada ao estado nutricional (Tabela 2). As gestantes com idades inferiores a 25 anos foram associadas a baixo peso e eutrofia e as com idades de 25 anos ou mais estavam associadas com sobrepeso e obesidade $(p=0,000)$. 
TABELA 2. Comparação das variáveis com o Estado Nutricional das gestantes.

\begin{tabular}{|c|c|c|c|c|c|c|c|c|c|c|}
\hline \multirow[b]{3}{*}{ Variável } & \multirow[b]{3}{*}{ Categoria } & \multicolumn{8}{|c|}{ Classificação } & \multirow[b]{3}{*}{$p^{*}$} \\
\hline & & \multicolumn{2}{|c|}{ Baixo Peso } & \multicolumn{2}{|c|}{ Eutrofia } & \multicolumn{2}{|c|}{ Obesidade } & \multicolumn{2}{|c|}{ Sobrepeso } & \\
\hline & & $\mathrm{N}$ & $\%$ & $\mathrm{~N}$ & $\%$ & $\mathrm{~N}$ & $\%$ & $\mathrm{~N}$ & $\%$ & \\
\hline \multirow[t]{5}{*}{ Faixa Etária } & Até 19 & 3 & 30,0 & 35 & 27,1 & 2 & 4,9 & 5 & 6,7 & 0,000 \\
\hline & $20-24$ & 7 & 70,0 & 30 & 23,3 & 7 & 17,1 & 23 & 30,7 & \\
\hline & $25-29$ & 0 & 0,0 & 31 & 24,0 & 10 & 24,4 & 20 & 26,7 & \\
\hline & $30-34$ & 0 & 0,0 & 24 & 18,6 & 13 & 31,7 & 16 & 21,3 & \\
\hline & Acima de 34 & 0 & 0,0 & 9 & 7,0 & 9 & 22,0 & 11 & 14,7 & \\
\hline \multirow{2}{*}{$\begin{array}{l}\text { Zona } \\
\text { moradia }\end{array}$} & Rural & 4 & 40,0 & 47 & 36,4 & 16 & 39,0 & 34 & 45,3 & 0,664 \\
\hline & Urbana & 6 & 60,0 & 82 & 63,6 & 25 & 61,0 & 41 & 54,7 & \\
\hline \multirow[t]{4}{*}{ Est Civil } & Solteira & 3 & 30,0 & 25 & 19,4 & 5 & 12,2 & 10 & 13,3 & 0,460 \\
\hline & Casada & 1 & 10,0 & 47 & 36,4 & 16 & 39,0 & 35 & 46,7 & \\
\hline & $\mathrm{C} /$ companheiro & 6 & 60,0 & 55 & 42,6 & 19 & 46,3 & 30 & 40,0 & \\
\hline & Outros & 0 & 0,0 & 2 & 1,6 & 1 & 2,4 & 0 & 0,0 & \\
\hline \multirow[t]{3}{*}{ Escolaridade } & Fundamental & 5 & 50,0 & 79 & 61,2 & 27 & 65,9 & 44 & 58,7 & 0,861 \\
\hline & Médio & 5 & 50,0 & 47 & 36,4 & 12 & 29,3 & 28 & 37,3 & \\
\hline & Superior & 0 & 0,0 & 3 & 2,3 & 2 & 4,9 & 3 & 4,0 & \\
\hline \multirow{3}{*}{$\begin{array}{l}\text { Renda } \\
\text { familiar }\end{array}$} & 1 ou $<1$ s.m. & 6 & 60,0 & 57 & 44,2 & 20 & 48,8 & 41 & 54,7 & 0,338 \\
\hline & 2 s.m. & 1 & 10,0 & 51 & 39,5 & 12 & 29,3 & 22 & 29,3 & \\
\hline & 3 ou $>$ s.m. & 3 & 30,0 & 21 & 16,3 & 9 & 22,0 & 12 & 16,0 & \\
\hline \multirow{2}{*}{$\begin{array}{l}\text { Classificação } \\
\text { Água }\end{array}$} & Insuficiente & 9 & 90,0 & 96 & 74,4 & 24 & 58,5 & 57 & 76,0 & 0,099 \\
\hline & Suficiente & 1 & 10,0 & 33 & 25,6 & 17 & 41,5 & 18 & 24,0 & \\
\hline \multirow{2}{*}{$\begin{array}{l}\text { Acrescenta + } \\
\text { sal comida }\end{array}$} & Sim & 0 & 0,0 & 14 & 10,9 & 5 & 12,2 & 13 & 17,3 & 0,342 \\
\hline & Não & 10 & 100,0 & 115 & 89,1 & 36 & 87,8 & 62 & 82,7 & \\
\hline \multirow{3}{*}{$\begin{array}{l}\text { Gordura } \\
\text { utilizada em } \\
\text { casa }\end{array}$} & Banha & 0 & 0,0 & 7 & 5,4 & 5 & 12,2 & 11 & 14,7 & 0,286 \\
\hline & Óleo & 8 & 80,0 & 107 & 82,9 & 30 & 73,2 & 54 & 72,0 & \\
\hline & Ambos & 2 & 20,0 & 15 & 11,6 & 6 & 14,6 & 10 & 13,3 & \\
\hline \multirow[t]{2}{*}{ Óleo } & Inadequada & 9 & 90,0 & 101 & 78,3 & 36 & 87,8 & 59 & 78,7 & 0,501 \\
\hline & Adequada & 1 & 10,0 & 28 & 21,7 & 5 & 12,2 & 16 & 21,3 & \\
\hline
\end{tabular}


TABELA 3. Comparação do consumo dietético com a recomendação da DRIs para gestantes.

\begin{tabular}{|c|c|c|c|c|c|c|c|}
\hline \multirow[b]{3}{*}{ Variável } & \multirow[b]{3}{*}{ Referência } & \multicolumn{6}{|c|}{ Classificação dos macro e micronutrientes } \\
\hline & & \multicolumn{2}{|c|}{ Insuficiente } & \multicolumn{2}{|c|}{ Adequado } & \multicolumn{2}{|c|}{ Acima } \\
\hline & & $\mathrm{N}$ & $\%$ & $\mathrm{~N}$ & $\%$ & $\mathrm{~N}$ & $\%$ \\
\hline VET & $36 \times \mathrm{IMC}+300 \mathrm{kcal}$ & 111 & 43,6 & 58 & 22,7 & 86 & 33,7 \\
\hline $\mathrm{CHO}$ & $45-65 \%$ & 23 & 9,0 & 212 & 83,2 & 20 & 7,8 \\
\hline PTN & $67-75 \mathrm{~g}$ & 51 & 20,0 & 25 & 9,8 & 179 & 70,2 \\
\hline LIP & $20-35 \%$ & 14 & 5,1 & 210 & 82,7 & 31 & 12,2 \\
\hline G Sat & Até $10 \%$ & - & - & 24 & 9,4 & 231 & 90,6 \\
\hline G Mono & $10-20 \%$ & 34 & 13,0 & 144 & 56,7 & 77 & 30,3 \\
\hline G Poli & $5-10 \%$ & 50 & 19,6 & 120 & 47,1 & 85 & 33,3 \\
\hline VIT A & $530^{*} / 550^{* *} \mu \mathrm{g}$ & 143 & 56,1 & 111 & 43,5 & 1 & 0,4 \\
\hline VIT C & $66^{*} / 70^{* *} \mathrm{MG}$ & 96 & 37,6 & 159 & 62,4 & - & - \\
\hline VIT D & $5 \mu \mathrm{g}$ & 228 & 89,5 & 20 & 7,8 & 7 & 2,7 \\
\hline Acido Fólico & $520 \mu \mathrm{g}$ & 254 & 99,6 & 0 & 0,0 & 1 & 0,4 \\
\hline Cálcio & $1300^{*} / 1000^{* *} \mathrm{mg}$ & 224 & 87,8 & 31 & 12,2 & - & - \\
\hline Ferro & $23^{*} / 22^{* *} \mathrm{MG}$ & 229 & 89,8 & 26 & 10,2 & - & - \\
\hline Zinco & $10,5^{*} / 9,5^{* *} \mathrm{mg}$ & 159 & 62,4 & 96 & 37,6 & - & - \\
\hline
\end{tabular}

*Referência para gestantes adolescentes. ${ }^{* *}$ Referência para gestantes adultas

A Tabela 3 mostra o comparativo entre as necessidades preconizadas e os resultados do consumo dietético encontrados. O consumo insuficiente de calorias foi prevalente em 43,5\% (111) das gestantes; $83,2 \%$ (212) apresentaram adequação na quantidade de carboidratos, $70,2 \%$ (179) um alto consumo de proteína e $82,7 \%$ (210) consumiam a quantidade adequada de lipídios. Observou-se um consumo abaixo do adequado de Vitamina A em 56,1\% (143); de Vitamina C em 37,6\% (96); de Vitamina D em 77,6\% (228); de Ácido Fólico em 99,6\% (254); de Cálcio em 87,8\% (224); de Ferro em 89,8\% (229) e de Zinco em 62,4\% (159). Os macronutrientes e micronutrientes não estavam significativamente associadas ao Estado Nutricional (Tabela 4). 
TABELA 4. Comparação consumo dietético com o Estado Nutricional das gestantes.

\begin{tabular}{|c|c|c|c|c|c|c|c|c|c|c|}
\hline \multirow[b]{3}{*}{ Variável } & \multirow[b]{3}{*}{ Categoria } & \multicolumn{8}{|c|}{ Classificação } & \multirow[b]{3}{*}{$p^{*}$} \\
\hline & & \multicolumn{2}{|c|}{\begin{tabular}{|l|} 
Baixo Peso \\
\end{tabular}} & \multicolumn{2}{|c|}{ Eutrofia } & \multicolumn{2}{|c|}{ Obesidade } & \multicolumn{2}{|c|}{ Sobrepeso } & \\
\hline & & $\mathrm{N}$ & $\%$ & $\mathrm{~N}$ & $\%$ & $\mathrm{~N}$ & $\%$ & $\mathrm{~N}$ & $\%$ & \\
\hline \multirow[t]{3}{*}{ VET } & Insuficiente & 4 & 40,0 & 48 & 37,2 & 23 & 56,1 & 36 & 48,0 & 0,386 \\
\hline & Adequado & 2 & 20,0 & 32 & 24,8 & 6 & 14,6 & 18 & 24,0 & \\
\hline & Acima & 4 & 40,0 & 49 & 38,0 & 12 & 29,3 & 21 & 28,0 & \\
\hline \multirow[t]{3}{*}{ Carboidrato } & Insuficiente & 1 & 10,0 & 8 & 6,2 & 3 & 7,3 & 11 & 14,7 & 0,609 \\
\hline & Adequado & 8 & 80,0 & 110 & 85,3 & 35 & 85,4 & 59 & 78,7 & \\
\hline & Acima & 1 & 10,0 & 11 & 8,5 & 3 & 7,3 & 5 & 6,7 & \\
\hline \multirow[t]{3}{*}{ Proteína } & Insuficiente & 1 & 10,0 & 25 & 19,4 & 13 & 31,7 & 12 & 16,0 & 0,383 \\
\hline & Adequado & 1 & 10,0 & 12 & 9,3 & 2 & 4,9 & 10 & 13,3 & \\
\hline & Acima & 8 & 80,0 & 92 & 71,3 & 26 & 63,4 & 53 & 70,7 & \\
\hline \multirow[t]{3}{*}{ Lipídios } & Insuficiente & 2 & 18,2 & 5 & 3,9 & 2 & 4,9 & 5 & 6,8 & 0,643 \\
\hline & Adequado & 7 & 63,6 & 107 & 82,9 & 37 & 90,2 & 59 & 79,7 & \\
\hline & Acima & 2 & 18,2 & 17 & 13,2 & 2 & 4,9 & 10 & 13,5 & \\
\hline Gordura & Insuficiente & 0 & 0,0 & 0 & 0,0 & 0 & 0,0 & 0 & 0,0 & 0,21 \\
\hline \multirow[t]{2}{*}{ Saturada } & Adequado & 1 & 10,0 & 14 & 10,9 & 5 & 12,2 & 4 & 5,3 & \\
\hline & Acima & 0 & 0,0 & 0 & 0,0 & 160 & 69,3 & 71 & 30,7 & \\
\hline Gordura & Insuficiente & 3 & 30,0 & 15 & 11,6 & 6 & 15,0 & 10 & 12,0 & 0,273 \\
\hline \multirow[t]{2}{*}{ Mono } & Adequado & 2 & 20,0 & 74 & 57,4 & 21 & 52,5 & 47 & 62,7 & \\
\hline & Acima & 5 & 50,0 & 40 & 31,0 & 13 & 32,5 & 19 & 25,3 & \\
\hline Gordura & Insuficiente & 1 & 10,0 & 23 & 17,8 & 10 & 24,4 & 16 & 21,3 & 0,108 \\
\hline \multirow[t]{2}{*}{ Poli } & Adequado & 7 & 70,0 & 52 & 40,3 & 20 & 48,8 & 41 & 54,7 & \\
\hline & Acima & 2 & 20,0 & 54 & 41,9 & 11 & 26,8 & 18 & 24,0 & \\
\hline \multirow[t]{3}{*}{ Vitamina A } & Insuficiente & 6 & 54,5 & 79 & 60,3 & 23 & 58,0 & 35 & 57,3 & 0,371 \\
\hline & Adequado & 5 & 45,4 & 51 & 38,9 & 16 & 42,0 & 39 & 42,7 & \\
\hline & Acima & 0 & 0,0 & 1 & 0,8 & 0 & 0,0 & 0 & 0,0 & \\
\hline \multirow[t]{2}{*}{ Vitamina C } & Insuficiente & 1 & 10,0 & 53 & 42,1 & 12 & 30,0 & 30 & 37,9 & 0,428 \\
\hline & Adequado & 9 & 90,0 & 73 & 57,9 & 28 & 70,0 & 49 & 62,1 & \\
\hline \multirow[t]{3}{*}{ Vitamina D } & Insuficiente & 8 & 88,9 & 116 & 89,9 & 36 & 83,7 & 68 & 91,9 & 0,655 \\
\hline & Adequado & 1 & 11,1 & 8 & 6,2 & 7 & 16,3 & 4 & 5,4 & \\
\hline & Acima & 0 & 0,0 & 5 & 3,9 & 0 & 0,0 & 2 & 2,7 & \\
\hline Ácido & Insuficiente & 10 & 100,0 & 129 & 100,0 & 41 & 100,0 & 74 & 98,7 & 0,470 \\
\hline Fólico & Adequado & 0 & 0,0 & 0 & 0,0 & 0 & 0,0 & 1 & 1,3 & \\
\hline \multirow[t]{2}{*}{ Cálcio } & Insuficiente & 8 & 80,0 & 117 & 90,7 & 38 & 92,7 & 61 & 81,3 & 0,167 \\
\hline & Adequado & 2 & 20,0 & 12 & 9,3 & 3 & 7,3 & 14 & 18,7 & \\
\hline
\end{tabular}




\begin{tabular}{|c|c|c|c|c|c|c|c|c|c|c|}
\hline \multirow[b]{3}{*}{ Variável } & \multirow[b]{3}{*}{ Categoria } & \multicolumn{8}{|c|}{ Classificação } & \multirow[b]{3}{*}{$p^{*}$} \\
\hline & & \multicolumn{2}{|c|}{ Baixo Peso } & \multicolumn{2}{|c|}{ Eutrofia } & \multicolumn{2}{|c|}{ Obesidade } & \multicolumn{2}{|c|}{ Sobrepeso } & \\
\hline & & $\mathrm{N}$ & $\%$ & $\mathrm{~N}$ & $\%$ & $\mathrm{~N}$ & $\%$ & $\mathrm{~N}$ & $\%$ & \\
\hline \multirow[t]{2}{*}{ Ferro } & Insuficiente & 8 & 100,0 & 116 & 90,6 & 36 & 78,3 & 69 & 94,5 & 0,579 \\
\hline & Adequado & 0 & 0,0 & 12 & 9,4 & 10 & 21,7 & 4 & 5,5 & \\
\hline \multirow[t]{2}{*}{ Zinco } & Insuficiente & 13 & 81,3 & 81 & 63,3 & 20 & 52,6 & 45 & 61,6 & 0,724 \\
\hline & Adequado & 3 & 18,7 & 47 & 36,7 & 18 & 47,4 & 28 & 38,4 & \\
\hline
\end{tabular}

Na Tabela 5, observou-se associação significativa entre o consumo de gordura saturada com o ganho de peso das gestantes $(p=0,013)$. O valor energético total (VET) e as demais variáveis não apresentaram relação significativa com a classificação de ganho de peso.

TABELA 5. Comparação do consumo dietético com o Ganho de peso das gestantes.

\begin{tabular}{|c|c|c|c|c|c|c|c|c|}
\hline \multirow[b]{3}{*}{ Variável } & \multirow[b]{3}{*}{ Categoria } & \multicolumn{6}{|c|}{ Classificação do ganho de peso } & \multirow[b]{3}{*}{$\mathrm{P}$} \\
\hline & & \multicolumn{2}{|c|}{ Baixo } & \multicolumn{2}{|c|}{ Adequado } & \multicolumn{2}{|c|}{ Acima } & \\
\hline & & $\mathrm{N}$ & $\%$ & $\mathrm{n}$ & $\%$ & $\mathrm{~N}$ & $\%$ & \\
\hline \multirow[t]{3}{*}{ VET } & Insuficiente & 14 & 46,7 & 42 & 44,2 & 55 & 42,3 & 0,842 \\
\hline & Adequado & 7 & 23,3 & 24 & 25,3 & 27 & 20,8 & \\
\hline & Acima & 9 & 30,0 & 29 & 30,5 & 48 & 36,9 & \\
\hline \multirow[t]{3}{*}{ Carboidratos } & Insuficiente & 3 & 10,0 & 9 & 9,5 & 11 & 8,5 & 0,974 \\
\hline & Adequado & 24 & 80,0 & 78 & 82,1 & 110 & 84,6 & \\
\hline & Acima & 3 & 10,0 & 8 & 8,4 & 9 & 6,9 & \\
\hline \multirow[t]{3}{*}{ Proteínas } & Insuficiente & 8 & 26,7 & 24 & 25,3 & 19 & 14,6 & 0,163 \\
\hline & Adequado & 1 & 3,3 & 8 & 8,4 & 16 & 12,3 & \\
\hline & Acima & 21 & 70,0 & 63 & 66,3 & 95 & 73,1 & \\
\hline \multirow[t]{3}{*}{ Lipídeos } & Insuficiente & 2 & 6,7 & 5 & 5,3 & 7 & 5,4 & 0,975 \\
\hline & Adequado & 26 & 86,7 & 78 & 82,1 & 106 & 81,5 & \\
\hline & Acima & 2 & 6,6 & 12 & 12,6 & 17 & 13,1 & \\
\hline Gordura & Insuficiente & 0 & 0,0 & 0 & 0,0 & 0 & 0,0 & 0,013 \\
\hline \multirow[t]{2}{*}{ Saturada } & Adequado & 6 & 20,0 & 16 & 16,8 & 2 & 1,5 & \\
\hline & Acima & 24 & 80,0 & 79 & 83,2 & 128 & 98,5 & \\
\hline Gordura & Insuficiente & 8 & 26,7 & 13 & 13,7 & 13 & 10,0 & 0,319 \\
\hline \multirow[t]{2}{*}{ Mono } & Adequado & 13 & 43,3 & 55 & 57,9 & 76 & 58,5 & \\
\hline & Acima & 9 & 30,0 & 27 & 28,4 & 41 & 31,5 & \\
\hline
\end{tabular}




\begin{tabular}{|c|c|c|c|c|c|c|c|c|}
\hline \multirow[b]{3}{*}{ Variável } & \multirow[b]{3}{*}{ Categoria } & \multicolumn{6}{|c|}{ Classificação do ganho de peso } & \multirow[b]{3}{*}{$\mathrm{P}$} \\
\hline & & \multicolumn{2}{|c|}{ Baixo } & \multicolumn{2}{|c|}{ Adequado } & \multicolumn{2}{|c|}{ Acima } & \\
\hline & & $\mathrm{N}$ & $\%$ & $\mathrm{n}$ & $\%$ & $\mathrm{~N}$ & $\%$ & \\
\hline Gordura & Insuficiente & 6 & 20,0 & 18 & 18,9 & 26 & 20,0 & 0,789 \\
\hline \multirow[t]{2}{*}{ Poli } & Adequado & 12 & 40,0 & 43 & 45,3 & 65 & 50,0 & \\
\hline & Acima & 12 & 40,0 & 34 & 35,8 & 39 & 30,0 & \\
\hline \multirow[t]{3}{*}{ Vitamina A } & Insuficiente & 8 & 26,7 & 55 & 57,9 & 80 & 61,5 & 0,280 \\
\hline & Adequado & 22 & 73,3 & 40 & 42,1 & 49 & 37,7 & \\
\hline & Acima & 0 & 0,0 & 0 & 0,0 & 1 & 0,8 & \\
\hline \multirow[t]{2}{*}{ Vitamina C } & Insuficiente & 18 & 60,0 & 32 & 33,7 & 46 & 35,4 & 0,130 \\
\hline & Adequado & 12 & 40,0 & 63 & 66,3 & 84 & 64,6 & \\
\hline \multirow[t]{3}{*}{ Vitamina D } & Insuficiente & 27 & 90,0 & 83 & 87,4 & 118 & 90,8 & 0,461 \\
\hline & Adequado & 3 & 10,0 & 7 & 7,4 & 10 & 7,7 & \\
\hline & Acima & 0 & 0,0 & 5 & 5,2 & 2 & 1,5 & \\
\hline \multirow[t]{2}{*}{ Acido Fólico } & Insuficiente & 30 & 100,0 & 95 & 100,0 & 129 & 99,2 & 0,485 \\
\hline & Adequado & 0 & 0,0 & 0 & 0,0 & 1 & 0,8 & \\
\hline \multirow[t]{2}{*}{ Cálcio } & Insuficiente & 28 & 93,3 & 86 & 90,5 & 110 & 84,6 & 0,342 \\
\hline & Adequado & 2 & 6,7 & 9 & 9,5 & 20 & 15,4 & \\
\hline \multirow[t]{2}{*}{ Ferro } & Insuficiente & 30 & 100,0 & 85 & 89,5 & 114 & 87,7 & 0,391 \\
\hline & Adequado & 0 & 0,0 & 10 & 10,5 & 16 & 12,3 & \\
\hline \multirow[t]{2}{*}{ Zinco } & Insuficiente & 10 & 59,4 & 60 & 65,2 & 89 & 61,1 & 0,584 \\
\hline & Adequado & 20 & 40,6 & 35 & 33,8 & 41 & 38,9 & \\
\hline
\end{tabular}

\section{Discussão}

No presente estudo, cerca da metade das gestantes apresentaram consumo insuficiente de calorias, resultado semelhante foi encontrado por Barros et al, (2004). Esse dado contrapõe a prevalência elevada de ganho de peso acima do recomendado (51\%) nas gestantes estudadas o que pode ser reflexo do subrelato característico de inquéritos dietéticos, constituindo uma limitação do método para avaliação do consumo alimentar (AVELINO et al, 2014).

Quanto aos macronutrientes, existe a recomendação de que aproximadamente 45 a $65 \%$ do valor energético total (VET) diário seja na forma de carboidratos (DRI, 2005). O atual estudo demonstrou que a maioria das gestantes relatou um consumo de carboidratos adequado, resultado semelhante encontrado no estudo de Nochieri et al. (2008).

No presente estudo constatou-se um consumo de proteína acima do preconizado, resultado semelhante foi verificado também em outros estudos 
(CARRUTH e SKINNER, 1991; HEDRICH et al., 2007). A demanda energéticoproteica aumentada está associada com a produção de novos tecidos e com o maior gasto energético, em função da massa corporal aumentada (CAMARGO e VEIGA, 2000).

O preconizado referente ao consumo de gorduras é 20 a 35\% do total do VET, sendo que menos de $10 \%$ deve ser de gordura saturada (DRI, 2005). No presente estudo, o consumo de lipídios da dieta na maioria das gestantes foi adequado, resultado semelhante a outros estudos, que também demonstraram com seu estudo um consumo adequado de lipídios (NOCHIERI et al., 2008; BUSCH et al., 2009). E quanto ao consumo de gordura saturada a maioria das gestantes apresentaram consumo acima do recomendado, semelhante ao estudo de Martins e Benício (2011), em que verificaram um maior consumo de gordura saturada ter relação com o ganho de peso elevado e retenção de peso após o parto.

O consumo de vitamina A neste estudo encontrou-se abaixo do adequado na maioria das gestantes no presente estudo, resultado semelhante ao de Bush et al. (2009), em que 77,77\% das gestantes também relataram baixa ingestão. A deficiência de vitamina A, pode causar cegueira noturna, associada com o risco de mortalidade materna cinco vezes maior nos dois anos pós-parto. (BUSCH et al., 2009).

Analisando o consumo de vitamina $\mathrm{C}$, o presente estudo demonstrou que mais da metade das gestantes consumiram a quantidade adequada, assim como no estudo de Azevedo e Sampaio (2003). O papel da vitamina C tem efeito positivo para redução de anemia, prevenção de pré-eclampsia e parto prematuro e tem correlação positiva com o peso ao nascer do bebê. Além disso, no estudo de Brognoli et al (2010), 63\% da gestantes apresentaram um consumo de vitamina $C$ abaixo do recomendado.

A maioria das gestantes apresentou a ingestão de vitamina $\mathrm{D}$ abaixo do recomendado, diferente do estudo de Baseggio (2012), que encontrou 48,7\% das gestantes com déficit no consumo de vitamina $\mathrm{D}$. A deficiência de vitamina $\mathrm{D}$ causa distúrbio da homeostase óssea na criança, e pode reduzir a mineralização óssea, aumentando o risco de fraturas (BASEGGIO, 2012).

O nível de ingestão de cálcio apresentou-se inferior ao recomendado na maioria das gestantes, resultado semelhante ao estudo de Barros (2004). O cálcio é fundamental para garantir a formação da estrutura óssea e dentária do feto, sua deficiência pode levar a retirada do cálcio dos ossos da mãe para suprir as necessidades de formação do feto, neste caso, as reservas de cálcio da mãe serão diminuídas, o que poderá causar osteoporose, perdas de dentes e cáries (ROCHA e JUNIOR, 2005).

A grande maioria das gestantes apresentou uma ingestão dietética de ácido fólico abaixo do recomendado, mas todas estavam fazendo uso de suplementação, cuja dose foi de $400 \mathrm{mg} /$ dia até a décima segunda semana 
gestacional. É importante ressaltar que a deficiência de ácido fólico pode prejudicar a divisão celular, causando defeito do tubo neural, aparecimento da anemia megaloblástica, prematuridade, aborto e recém-nascidos que apresentam deficiências congênitas (BROGNOLI et al., 2010).

Quanto ao consumo dietético de ferro do presente estudo, a maioria das gestantes apresentaram um consumo abaixo do recomendado, assim como os resultados de outros estudos observaram baixa adequação (AZEVEDO e SAMPAIO 2003). Todas gestantes estavam fazendo uso de suplementação de ferro o que pode amenizar os riscos. A ingestão de zinco também aparece abaixo do preconizado em um pouco mais da metade das gestantes, corroborando com o estudo de Nogueira, et al. (2003) onde demonstraram que $80 \%$ estavam abaixo da adequação de zinco.

No presente estudo a relação do estado nutricional das gestantes com a idade mostrou-se significativa de forma que as gestantes com 25 anos ou mais apresentaram maior prevalência de excesso de peso, o que se assemelha ao estudo de Gomes et al. (2014). Parte do ganho de peso em excesso pode ser em resposta ao consumo de gordura saturada, pois foi verificada associação significativa entre o consumo adequado e em excesso de gordura saturada com o ganho de peso das gestantes. O consumo calórico e dos demais nutrientes estudados não apresentou associação estatisticamente significativa com o ganho de peso gestacional, mas isso pode não representar ausência de associação. Pois caso fossem realizados um maior número de repetições do R24h em parte da amostra, o erro aleatório poderia ser reduzido e a precisão da estimativa aumentada (VERLY-JR et al, 2018).

\section{Conclusão}

Conclui-se que o ganho de peso gestacional pode ter influência direta do consumo de alimentos ricos em gordura saturada. Esse achado representa a necessidade de adequação da dieta das gestantes evidenciando a importância de intervenções nutricionais individualizadas e coletivas no sentido de prevenir agravos à saúde das gestantes e seus conceptos.

\section{Referências}

AVELINO G. F., PREVIDELLI A. N., DE CASTRO M.A., MARCHIONI D. M. L., FISBERG R. M. Sub-relato da ingestão energética e fatores associados em estudo de base populacional. Caderno de Saúde Pública, 30(3):663-68, 2014.

AZEVEDO D. V., SAMPAIO H. A. C. Consumo alimentar de gestantes adolescentes atendidas em serviço de assistência pré-natal. Revista de Nutrição de Campinas, 16(3):273-280, 2003. 
BARROS D. C., PEREIRA R. A., GAMA S. G. N., LEAL M. C. O consumo alimentar de gestantes adolescentes no Município do Rio de Janeiro. Caderno de Saúde Pública, 20 Sup 1:S121-S129, 2004.

BASEGGIO M. H. Avaliação do consumo alimentar de gestantes portadoras de diabetes pré-gestacional pelo método do recordatório alimentar de 24 horas [dissertação]. São Paulo: Faculdade de medicina da Universidade de São Paulo, Programa de Obstetrícia e Ginecologia, 2012.

BROGNOLI A. F., NEME L. C. L. H., PASSONI C. M. S. Relação da dieta de gestantes com o estado nutricional. Cadernos da Escola de Saúde Curitiba, 03: 1-14. 2010.

BUSCH L. I., SILVA A. C. P., BOSCO S. M. D. Adequação do consumo alimentar das gestantes freqüentadoras de um grupo de gestantes de um município do interior do Rio Grande do Sul. Caderno Pedagógico, 6(2): 9-28. 2009.

CAMARGO R. M. S., VEIGA G. V. Ingestão e hábitos alimentares de adolescentes gestantes. A Folha Médica, 119(3):37 - 46, 2000.

CARRUTH B. R., SKINNER J. D. Pregnant adolescents report infrequent use of sugar substitutes. Journal American Dietetic Association, 91(5):608-10, 1991

DA SILVA L. S. V., THIAPÓ A. P., DE SOUZA G. G., SAUNDERS C., RAMALHO A. Micronutrientes na gestação e lactação. Revista Brasileira de Ginecologia e Obstetrícia, 7(3):237-244, 2007.

Dietary Reference Intakes for Energy, Carbohydrate, Fiber, Fat, Fatty Acids, Cholesterol, Protein, and Amino Acids (Macronutrients). Washington, D. C.: National Academic Press, 2005, p. 1331.

Dietary Reference Intakes for Thiamin, Riboflavin, Niacin, Vitamin B6, Folate, Vitamin B12, Pantothenic Acid, Biotin, and Chroline. Washington, D. C.: National Academic Press, 1998, p. 567.

FAZIO E. S., NOMURA R. M. Y., DIAS M. C. G., ZUGAIB M. Consumo dietético de gestantes e ganho ponderal materno após aconselhamento nutricional. Revista Brasileira de Ginecologia e Obstetrícia, 33(2):87-92, 2011.

GIACOMELLO A., SCHMIDT M. I., NUNES M. A. A., DUNCAN B. B., SOARES R. M., MANZOLLI P., CAMEY S. Validação relativa de Questionário de Frequencia Alimentar em gestantes usuárias de serviços do Sistema Único de Saúde em dois municípios no Rio Grande do Sul, Brasil. Revista Brasileira Saúde Materno Infantil, 8(4):445-545, 2008.

GOMES R. N. S., GOMES V. T. S. CALDAS D. R. C., LAGO E. C., CAMPOS F. K. L. C., GOMES M. S. Sub-relato da ingestão energética e fatores associados em estudo de base populacional. Revista Interdisciplinar, 7(4):81-90, 2014. 
HEDRICH A., NOVELLO D., RUVIARO L., ALVES J., QUINTILIANO D.A. Perfil alimentar, estado nutricional, de saúde e condições sócio-econômicas de gestantes assistidas por centros de saúde do município de Guarapuava-PR. Revista SalusGuarapuava-PR, 1(2):139-146, 2007.

Institute of Medicine. Subcommittee on Nutritional Status and Weight Gain During Pregnancy. Nutrition during pregnancy: part I, weight gain; part II, nutrient supplements. Washington, Dc: National Academy Press, 1990.

MARTINS A. P. B., BENICIO M. H. A. Influência do consumo alimentar na gestação sobre a retenção de peso pós-parto. Revista de Saúde Pública, 45(5):870-77, 2011.

MELO A. S. O., ASSUNÇÃO P. L., GONDIM S. S. R., CARVALHO D. F., AMORIM M. M. R., BENICIO M. H. D. A., CARDOSO M. A. A. Estado Nutricional Materno, ganho de peso gestacional e peso ao nascer. Revista Brasileira de Epidemiologia, 10(2): 249$57,2007$.

NOCHIERI, A. C. M., ASSUMPÇÃO M. F., BELMONTE F. A. L., LEUNG M. C. A. Perfil nutricional de gestantes atendidas em primeira consulta de nutrição no prénatal de uma instituição filantrópica de São Paulo. O Mundo da Saúde São Paulo, 32(4):443-451, 2008.

NOGUEIRA, N. N., PARENTE, J. V., COZZOLINO, S. M. F. Mudanças na concentração plasmática de zinco e acido fólico em adolescentes grávidas submetidas a diferentes esquemas de suplementação. Caderno de Saúde Pública, 19(1):155-160, 2003.

NRC. National Research Council. Recommended Dietary Allowances. 10ed. Washington: National Academy Press, p.284, 1989.

ROCHA F. A. C., JUNIOR F. S. S. Osteoporose e Gravidez. Revista Brasileira de Reumatologia, 45(3): 141-145, 2005.

WHO. World Health Organization. Diet, nutrition and the prevention of chronic diseases. Geneva, (Technical Report Series 797), 1990.

VERLY-JR E., SICHIERI R., BALTAR V. T. Correção de medidas de associação pela variação do dia a dia no consumo alimentar: avaliação do desempenho por meio de simulação. Cadernos de Saúde Pública, 33():e00173216, 2017. 